\title{
Fractional Chern Insulator
}

\author{
N. Regnault ${ }^{1}$ and B. Andrei Bernevig ${ }^{2}$ \\ ${ }^{1}$ Laboratoire Pierre Aigrain, ENS and CNRS, 24 rue Lhomond, 75005 Paris, France \\ ${ }^{2}$ Department of Physics, Princeton University, Princeton, New Jersey 08544, USA
}

(Received 16 June 2011; published 2 December 2011)

\begin{abstract}
Chern insulators are band insulators exhibiting a nonzero Hall conductance but preserving the lattice translational symmetry. We conclusively show that a partially filled Chern insulator at $1 / 3$ filling exhibits a fractional quantum Hall effect and rule out charge-density-wave states that have not been ruled out by previous studies. By diagonalizing the Hubbard interaction in the flat-band limit of these insulators, we show the following: The system is incompressible and has a 3-fold degenerate ground state whose momenta can be computed by postulating an generalized Pauli principle with no more than 1 particle in 3 consecutive orbitals. The ground-state density is constant, and equal to $1 / 3$ in momentum space. Excitations of the system are fractional-statistics particles whose total counting matches that of quasiholes in the Laughlin state based on the same generalized Pauli principle. The entanglement spectrum of the state has a clear entanglement gap which seems to remain finite in the thermodynamic limit. The levels below the gap exhibit counting identical to that of Laughlin 1/3 quasiholes. Both the 3 ground states and excited states exhibit spectral flow upon flux insertion. All the properties above disappear in the trivial state of the insulator-both the many-body energy gap and the entanglement gap close at the phase transition when the single-particle Hamiltonian goes from topologically nontrivial to topologically trivial. These facts clearly show that fractional many-body states are possible in topological insulators.
\end{abstract}

DOI: 10.1103/PhysRevX.1.021014 Subject Areas: Condensed Matter Physics, Strongly Correlated Materials, Topological Insulators

\section{INTRODUCTION}

The interest in the field of topological insulators has exploded in recent years, fueled by the theoretical prediction $[1,2]$ and experimental observation $[3,4]$ of the topological insulators that preserve the time-reversal symmetry. These insulators have an insulating bulk but exhibit perfectly metallic edges or surface states whose existence is required by the nontrivial topology of the bulk electronic structure. However, most of the theoretical work on topological insulators is essentially of a single-particle nature and neglects interactions, except for perhaps at randomphase-approximation level. The few interacting states [5] that have been proposed to give rise to topological insulators can be understood from a mean-field perspective [6]their quasiparticle excitations are still electronlike and not anyonlike. As such, the type of interacting topologically ordered states with fractional-statistic quasiparticles, such as the fractional quantum Hall $(\mathrm{FQH})$ states, has not been found or proposed in an interacting topological insulator. The discovery of topologically ordered states of matter in the absence of the usual external magnetic field applied to the system would be of tremendous interest to the condensed-matter community and could potentially revolutionize fields such as topological quantum computation.

Published by the American Physical Society under the terms of the Creative Commons Attribution 3.0 License. Further distribution of this work must maintain attribution to the author(s) and the published article's title, journal citation, and DOI.
Interestingly, it has recently been suggested [7-10] that a Chern insulator, i.e., a zero magnetic equivalent of the quantum Hall effect [11], with strong interactions (compared to the bandwidth) has as a ground state, a fractional Hall effect at filling $1 / 3$ as its ground state. By diagonalizing the Hubbard interaction in a model with an almostflat-band dispersion, the authors of [7-9] have found, for small sizes of the system, an almost 3-fold degenerate ground state, reminiscent of the fractional quantum Hall state on a torus. However, the origins of such a degenerate state can be either a charge-density wave or a fractional quantum Hall state. Arguments were given for why the state should be a FQH state, but they do not differentiate qualitatively between the FQH state and a charge-density wave, as will be shown later. The very small sizes of the systems considered in the previous works and the lack of qualitatively unequivocal calculations that prove the existence of a fractional Chern insulator state make such a state still elusive. In the present paper, we present the proof of principle that a $\nu=1 / 3 \mathrm{FQH}$ state exists in the nontrivial Chern insulator subject to a Hubbard interaction.

By working in the flat-band limit where the kinetic energy is zero and the band gap can be set to infinity, we present multiple results that strongly suggest the presence of a $\nu=1 / 3 \mathrm{FQH}$ state. We show that a repulsiveinteraction Hamiltonian has a 3-fold degenerate ground state at filling $1 / 3$ separated from the excited states by a gap, which we show approaches a finite value in the thermodynamic limit. Upon the adiabatic insertion of a magnetic flux quantum, the degenerate ground states flow 
into each other-and cross without level repulsion at large particle number. The flux insertion and the 3 -fold groundstate degeneracy have already been found in two earlier papers, but, without proving the existence of a finite gap in the thermodynamic limit, they could very well be the hallmark of a charge-density wave (CDW). By going to larger sizes than previously achieved, we show that the energy gap between the 3-fold degenerate ground state and the first excited states seems to remain finite in the thermodynamic limit when the single-particle Hamiltonian is a nontrivial Chern insulator. Indeed, a commensurate CDW at a fractional filling would also give rise to a gaped phase. Thus, the gap argument might be insufficient to prove the existence of a Laughlin-like phase. The ground states occur at lattice momenta consistent with an emergent generalized Pauli principle forbidding the presence of more than 1 particle in 3 consecutive orbitals. We compute the momentum-space density $n(\vec{k})$ of each of the 3 degenerate ground states and find it to be a constant approaching $1 / 3$ for all $\vec{k}$, another clue that the ground state is not a CDW but a true $\mathrm{FQH}$ state. We then show that the quasihole excitations of these states resemble Laughlin-FQH excitations: Their total counting matches exactly that of the Laughlin quasihole states on the torus. We also present a heuristic Pauli principle which, in some cases, counts the number of quasihole states for each momentum sector. The matching of the excitation properties is a clear signal that the state observed is a FQH state: Charge-density-wave states would not have an excitation spectrum resembling that of a FQH state. We then compute the entanglement spectrum (ES) for the ground state of the repulsiveinteraction Hamiltonian, find a large entanglement gap, and show that the counting of the entanglement states below the gap matches that of Laughlin quasiholes. The entanglement gap remains finite in the thermodynamic limit, and closely tracks the gap in the energy spectrum as the system is tuned through a phase transition of the single-particle Hamiltonian between the nontrivial Chern insulators and a trivial band insulator. We also discuss the dependence of the state on the symmetries and aspect ratio of the lattice and on the parameters of the Chern insulators. Both the energy spectrum and the entanglement spectrum of the interacting system change fundamentally as the single-particle Chern-insulator Hamiltonian undergoes a phase transition and becomes a trivial band insulator. In the end, we discuss the analytical principle behind the counting of quasihole states on the lattice.

\section{THE MODEL AND ITS SYMMETRIES}

Several Chern-insulator models with an approximate flat band have been recently proposed $[8,10,12]$. For our model, we pick the Chern insulator on a checkerboard lattice, first introduced in $[8,12]$. This model already exhibits weak dispersion of the bands, but because we work in the flatband limit, this is not essential to our calculation-we could have picked an alternate single-particle Hamiltonian. As written in [12] the one-body Hamiltonian reads $H_{1}=\sum_{k}\left(c_{k A}^{\dagger}, c_{k B}^{\dagger}\right) h_{1}(k)\left(c_{k A}, c_{k B}\right)^{T}$ with $A, B$ being the two sites in the unit cell. The Hamiltonian matrix can be expressed in terms of the 3 Pauli matrices $h_{1}(k)=$ $\sum_{i} d_{i}(k) \sigma_{i}$ where the $d_{i}(k)$ 's are $d_{x}(k)=4 t_{1} \cos (\phi) \times$ $\cos \left(k_{x} / 2\right) \cos \left(k_{y} / 2\right), \quad d_{y}(k)=4 t_{1} \sin (\phi) \sin \left(k_{x} / 2\right) \times$ $\sin \left(k_{y} / 2\right), \quad d_{z}=2 t_{2}\left[\cos \left(k_{x}\right)-\cos \left(k_{y}\right)\right]+M$. In the original model [12] there is an additional diagonal term$4 t_{3} \cos \left(k_{x}\right) \cos \left(k_{y}\right)$-which is of course relevant for the energy (and shrinks the dispersion of the bands, thereby making them flatter) but does not matter for the eigenstates. Since we are diagonalizing in the flat-band limit, we neglect this term. $\phi$ is the phase factor added to the nearestneighbor hoppings, while the parameter $M$ is a mass added in order to drive the transition from a topological Chern insulator (for $M=0$ ) to a trivial atomic-limit insulator when $M \rightarrow \pm \infty$. The model always has an energy gap (for $t_{1}, t_{2}, \phi$ not vanishing) with the exception of the points $k_{x}=0, k_{y}=\pi, M=-4 t_{2}$, and $k_{x}=\pi, k_{y}=0, M=4 t_{2}$, where a gap is absent and where the phase transitions between the atomic limits $M \rightarrow \pm \infty$ and the Cherninsulator phase occur. For $|M|<4\left|t_{2}\right|$, the filled valence band has a Hall conductance of 1 . The single-particle Hamiltonian matrix has the following symmetries: inversion with identity inversion matrix $h_{1}\left(-k_{x},-k_{y}\right)=$ $h_{1}\left(k_{x}, k_{y}\right)$, as well as (at $M=0$ ) a certain type of particlehole symmetry coupled with a $C_{4}$ rotation and a mirror operation, $\sigma_{z} h_{1}\left(k_{x}, k_{y}\right) \sigma_{z}=-h_{1}\left(k_{y}, k_{x}\right)$. Unfortunately, due to the presence of fractions $k / 2$, the model in [12] is not in Bloch form. To render it in Bloch form, we perform the gauge transformation $c_{k B} \rightarrow c_{k B} \exp \left[-i\left(k_{x}-k_{y}\right) / 2\right]$ to obtain

$$
h_{2}(k)=\left(\begin{array}{cc}
h_{11}(k) & h_{12}(k) \\
h_{12}^{\star}(k) & -h_{11}(k)
\end{array}\right),
$$

where $h_{11}(k)=2 t_{2}\left[\cos \left(k_{x}\right)-\cos \left(k_{y}\right)\right]+M$,

$$
h_{12}(k)=t_{1} e^{i \phi}\left[1+e^{i\left(k_{y}-k_{x}\right)}\right]+t_{1} e^{-i \phi}\left(e^{i k_{y}}+e^{-i k_{x}}\right) .
$$

The inversion symmetry of $h_{1}(k)$ translates into another symmetry of $h_{2}(k)$ given by $U^{\dagger}(k) h_{2}(\vec{k}) U(k)=h_{2}(-\vec{k})$ with $U(k)$ being a diagonal $2 \times 2$ unitary matrix with 1 , $e^{-i\left(k_{x}-k_{y}\right) / 2}$ on the diagonal. We now fractionally fill the valence band of this insulator and add interactions. The existence of a FQH state in a Chern insulator should not be taken for granted. Unlike the Landau level, a band insulator has nonzero bandwidth and a Berry-phase distribution, which cannot be made uniform over the full Brillouin zone. Fractionally filling the band will, even in the presence of large interactions, allow the particles to cover only part of the Brillouin zone and hence feel only part of the Berry curvature. To eliminate the effect of the band curvature, and to allow the filled particles to 
democratically sample the whole Brillouin zone, we always work in the flat-band limit of a topological insulator. This corresponds to keeping the single-particle eigenstates of $h_{2}(k)$ but putting the energies of the occupied bands to be an arbitrary energy $\pm E_{0}$ where $E_{0}>0$. At the Hamiltonian level, we transform from $h_{2}(k)=E_{-}(k) P_{-}(k)+$ $E_{+}(k) P_{+}(k)$ to $h_{2}^{F B}(k)=-E_{0} P_{-}(k)+E_{0} P_{+}(k)$, where $P_{ \pm}$are the projectors onto the occupied and unoccupied bands. As such, the energy difference between the valence and conduction bands can be made large without changing the eigenstates of the system. We diagonalize the interaction Hamiltonian directly in the filled band, neglecting the conduction band. This is similar to the lowest-Landau-level (LLL) projection in the usual fractional quantum Hall effect. A nice feature of the checkerboard-lattice model is that the Hubbard interaction is fixed and has a simple form:

$$
H_{\text {interaction }}=\sum_{\langle i j\rangle} n_{i} n_{j},
$$

where $i, j$ are nearest-neighbor sites. One can see that the interaction only couples $A$ with $B$ sites. This is not the case for multiorbital models, in which case there is both an on-site and nearest-neighbor interaction. Upon Fourier transform and gauge transformation, the interaction reads

$$
\frac{1}{N} \sum_{k_{1,2,3,4}} \delta_{\vec{k}_{1}+\vec{k}_{3}-\vec{k}_{2}-\vec{k}_{4} ; \bmod 2 \pi} V_{k_{1}, k_{2}, k_{3}, k_{4}} c_{k_{1} A}^{\dagger} c_{k_{2} A} c_{k_{3} B}^{\dagger} c_{k_{4} B},
$$

where

$$
V_{k_{1}, k_{2}, k_{3}, k_{4}}=\left[1+e^{i\left(k_{4 y}-k_{3 y}\right)}\right]\left[1+e^{-i\left(k_{4 x}-k_{3 x}\right)}\right] .
$$

In passing, we remark that the present interaction and matrix elements are, in principle, very different from the pseudopotential Hamiltonians that give rise to the fractional quantum Hall effect in the lowest Landau level.

\section{NUMERICAL PROCEDURE AND RESULTS}

We now diagonalize this Hamiltonian for $N$ particles in a $N_{x} \times N_{y}$ lattice, where, for the ground state we have $N_{x} \cdot N_{y}=3 N$ (we concentrate on the $\nu=1 / 3$ filling), while for the quasihole excitations we have $N_{x} \cdot N_{y}>$ $3 N$. This setup is quite different from the fractional quantum Hall effect on a lattice [13-15], where the lattice size can be changed while keeping fixed both $N$ and $\nu$. In our case, only the lattice aspect ratio might be tuned in some cases (not all of them are accessible for a given $N$ and $\nu$ ). All the numerical calculations are performed with $t_{2}=$ $(2-\sqrt{2}) / 2 t_{1}$ as discussed in [12]. The total translation operators in the $x, y$ directions commute with both the single and many-body Hamiltonians and hence the eigenstates are indexed by total momentum quantum numbers $\left(K_{x}, K_{y}\right)$, which are the sum of the momentum quantum numbers of each of the $N$ particles modulo $\left(N_{x}, N_{y}\right)$. The basis states are $\prod_{i=1}^{N} \gamma_{-, \vec{k}_{1}}^{\dagger} \ldots \gamma_{-, \vec{k}_{N}}^{\dagger}|0\rangle$ (we work in the
LLL, and the $\gamma_{-, \vec{k}}^{\dagger}$ 's are the creation operators for a particle of momentum $\vec{k}$ in the valence band). When acting on the basis states, $c_{\vec{k}, \alpha}=u_{-, \alpha, \vec{k}} \gamma_{-, \vec{k}}$, where $u_{-, \alpha, \vec{k}}$ is the $\alpha=$ $A, B$ component of the eigenstate of the occupied band of $h_{2}(k)$ or $h_{2}^{F B}(k)$ (they have identical eigenstates). Diagonalizing directly in the valence band provides for large numerical efficiency. The inversion symmetry of the single-particle problem is maintained at the level where the many-body interaction is taken into account. Thus, the spectrum has an exact $\left(K_{x}, K_{y}\right) \rightarrow\left(-K_{x},-K_{y}\right)$ symmetry which can be used as checkup. We first perform all the calculations for the $M=0$ nontrivial Chern insulator, and then drive the fractional Chern insulator to a phase transition by increasing $M$.

\section{A. Degenerate ground state and its properties}

In Fig. 1 we show the spectrum of the system for several sizes $N=6,10,12$ and aspect ratios $\left(N_{x}, N_{y}\right)=(3,6)$, $(5,6),(6,6)$ in the $M=0$ nontrivial Chern-insulator case. The choice of aspect ratios will be explained later. We observe a 3-fold degenerate ground state at lattice momenta $\{(0,3),(0,3),(0,3)\}, \quad\{(0,1)(0,3)(0,5)\}$, and $\{(0,0),(0,0)(0,0)\}$. The principle that determines these momenta will be explained later. The insertion of flux quantum in either of the $x, y$ directions can be performed by letting each single-particle momentum $k_{x, y} \rightarrow k_{x, y}+$ $\Phi / N_{x, y}$ with $\Phi \in[0,2 \pi]$. For the case where $N=10$, Fig. 2 shows that the 3 degenerate states (which occur at the same lattice momentum and are hence split by the interaction) experience spectral flow into each other (as also shown previously in Ref. [8]). In the presence of a gap, 3 -fold degenerate ground states that experience spectral flow in a $\nu=1 / 3$ fractionally filled Chern insulator are enough to guarantee the presence of a $\sigma_{x y}=1 / 3$ fractional quantum Hall state. Upon the insertion of 3 fluxes, the ground states move back to the initial configuration, but, as the filled band has Chern number unity, one electron has

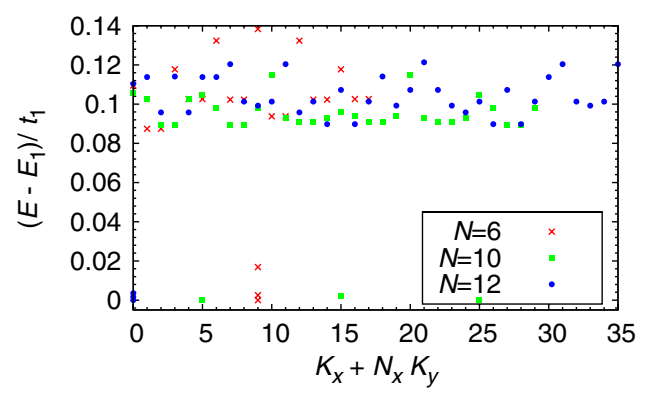

FIG. 1. Low-energy spectrum for $N=6,10$, and 12, $N_{x}=$ $N / 2, N_{y}=6$. The energies are shifted by $E_{1}$, the lowest energy for each system size. We only show the lowest energy per momentum sectors in addition to the 3 -fold ground state. We note the good ground-state degeneracy, even for relatively small system sizes such as 6 particles. 


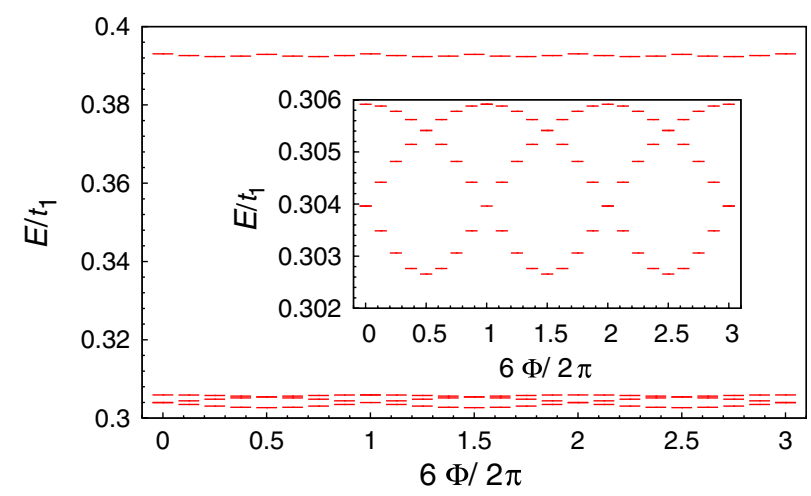

FIG. 2. Evolution of the 3-fold degenerate ground state upon flux insertion along the $y$ direction at $N=10, N_{x}=5$, and $N_{y}=6$. The 3-fold degenerate ground-states spectral flow into each other (inset) separated at each point in the flux insertion from the first excited state (the only one of the excited states shown here), which does not exhibit spectral flow with any of the other states.

been transferred across the gap, giving a Hall conductance of $\sigma_{x y}=1 / 3$. These arguments have already been presented in $[7,8]$ but they are valid only in the presence of a spectral gap, which was not proved in $[7,8]$. In the absence of such a proof, the effects presented in $[7,8]$ could also occur in a $1 / 3$ charge-density wave. The existence of a gap in the thermodynamic limit is complicated by commensuration effects on the lattice. Because of the system sizes that can be accessed numerically, we cannot give a quantitative value for the size of the gap in the thermodynamic limit. Our results do, however, strongly suggest that this value should be finite.

The finite-size scaling of the gaps $\Delta$ is presented in Fig. 3. Several crucial trends are visible. For $N_{y}=3$, the gap is finite but decreasing as we increase $N$ (or equivalently $N_{x}=3 N / N_{y}$ ). Its decrease does not mean that the $\mathrm{FQH}$ state is compressible in the thermodynamic limit. Indeed, the thermodynamic limit here does not correspond to a 2-dimensional system: for $N \rightarrow \infty$, if $N_{y}$ is kept fixed at 3 , we reach the case of a one-dimensional system. This

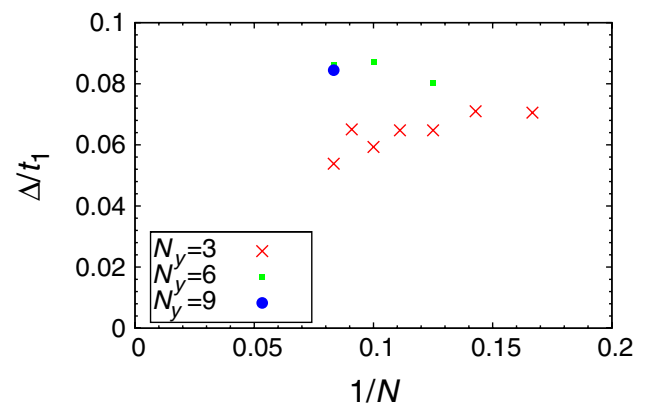

FIG. 3. Energy gap $\Delta$ for different system sizes and aspect ratio. The gap is defined as the difference between the energy of the first excited state and the highest energy of the 3-fold groundstate manifold. In each case, $N_{x}=3 N / N_{y}$.

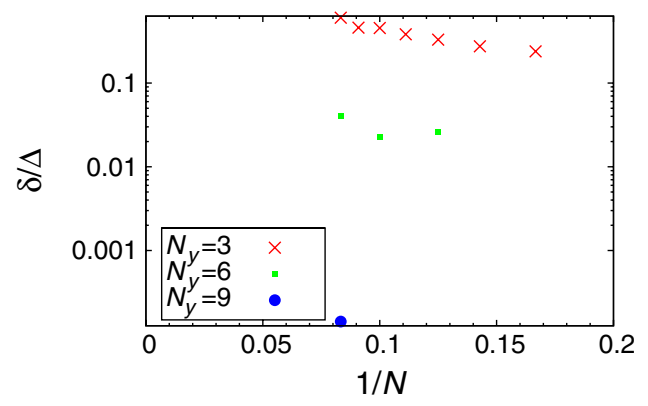

FIG. 4. Ratio between the energy spread $\delta$ of the 3-fold ground-state manifold and the energy gap $\Delta$ for various system sizes and aspect ratio. The spread is defined as the difference between the highest energy and the lowest energy of the 3-fold ground-state manifold. In each case, $N_{x}=3 N / N_{y}$.

system, even at the single-particle level, cannot have a Chern number-as discussed later, Chern numbers appear only in the true 2-dimensional limit. As such, it is expected that, for fixed $N_{y}$, the gap diminishes in the thermodynamic limit. Going to $N_{y}=6$ shows a large increase in the gap from $N_{y}=3$ exactly because the aspect ratio changed and the system is more 2 dimensional. However, if we keep $N_{y}$ fixed, the gap will also start diminishing as we go to the thermodynamic limit, a fact clearly shown in Fig. 3. When we go to larger $N_{y}$ values, the gap increases again. We are thus confident that the gap remains open and scales to a finite value for $N_{x} / N_{y} \rightarrow$ finite, $N \rightarrow \infty$.

The energy spread is plotted in Fig. 4. If $E_{1}, E_{2}, E_{3}$ are the energies of the 3 quasidegenerate ground states and $E_{4}$ is the energy of the first state above the lowest 3 states, then the spread $\delta=E_{3}-E_{1}$ while the gap $\Delta=E_{4}-E_{3}$. Figure 4 the degree of degeneracy relative to the size of the gap as a function of $N=N_{x} \cdot N_{y} / 3$ for fixed $N_{y}$. We see that the degeneracy gets better and better for larger $N_{y}$.

We have also plotted the momentum-space density of the 3 degenerate states in the fractional Chern insulator. In a $\mathrm{FQH}$ state on the sphere or on the torus, the occupation of every angular-momentum orbital is constant and equal to $1 / 3$ (the filling factor) in the thermodynamic limit. In the

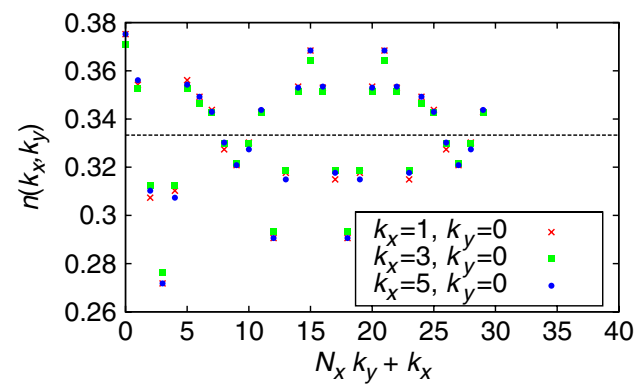

FIG. 5. Occupation number of each of the single-particle momentum orbitals for the three $N=10$ ground states (which occur at different total momenta) at $N_{x}=5, N_{y}=6$. Notice that the occupation number is uniform and close to the filling factor $1 / 3$. 
current case, we expect in finite-size deviations from the constant-density $1 / 3$ scenario, due to the fact that the Berry curvature is not uniform in the Brillouin zone. We, however, also expect that the deviations not be very large because, in the end, the states must be an incompressible liquid. The standard deviation of the momentum-space density strongly depends on the aspect ratio, typically $\sim 0.1$ for $N_{y}=3$ and $\sim 0.02$ for $N_{y}=6$ (almost independent from $N$ ). The results are presented in Fig. 5. We observe that the occupation numbers of each of the momenta of the 3 degenerate ground states for the $N=10$ particle state are close to $1 / 3$, as expected for an incompressible liquid at this filling. As the 3 ground states occur at different momenta, the fact that each of the ground states has a uniform $n(k)$ close to $1 / 3$ for any value of $k$ removes the possibility that these states are charge-density waves.

\section{B. Quasihole excitations}

To iron out any doubt about the existence of a FQH state, we now investigate the quasihole excitations of the system. The excitations of a FQH state are very specific in the sense that they carry a fractional $1 / 3$ charge and exhibit fractional $1 / 3$ statistics. This would not happen in a charge-density-wave state. The quasihole excitations arise by inserting a flux in the FQH state. These properties endow the excitations with a state counting specific to the FQH universality class at a certain filling, independent of the specific Hamiltonian to be diagonalized or even of the form of the model wave function. In the current case, it is expected that the actual form of the ground-state wave function is not of a simple known Laughlin form. However, it is also expected that the FQH at filling $1 / 3$ is the Abelian FQH 1/3 state, whose universal properties (after integrating out the fermions) are described by the topological Abelian Chern-Simons field theory. As such, its universal quasihole counting (even in finite size) should be identical to that of the usual Laughlin quasihole states. In fact, as our interaction represents a generic Hamiltonian (similar to the Coulomb Hamiltonian in the $\mathrm{FQH}$ case), all we can hope for is that the set of quasiholes is separated by a finite gap from higher-energy nonuniversal excitations that are not described by the topological field theory.

The FQH $\nu=1 / 3$ quasihole counting is identical to that of particles with Haldane $1 / 3$ statistics in both the thermodynamic limit and in the finite-size systems. This counting can be directly obtained from the topological ChernSimons field theory and the assumption of bulk-edge correspondence: In a system with the boundary, the Abelian $1 / \nu$ Chern-Simons field theory is not gauge invariant, it is missing an edge piece which is a boson at compactification radius $\sqrt{1 / \nu}$. The modes of this boson are the edge modes, a gapless conformal field theory whose excitations, when placed in a finite-size box, exhibit Haldane $1 / \nu$ statistics: Every particle must be separated from another particle by at least $1 / \nu$ orbitals. This is called the $(1,1 / \nu)$ generalized Pauli principle, which will be presented in the next section. However, the bulk-edge correspondence renders this counting also the same as that of the quasiholes in the bulk of the FQH state, and represents a hallmark of the $\nu=1 / 3 \mathrm{FQH}$ state.

We now compare such a counting with that obtained from numerics. For the model FQH Hamiltonians such as the Haldane pseudopotentials, the quasiholes are just the degenerate ground states of the system when fluxes are added to the system. In this sense, the ground state is just the highest-density state of a set of other degenerate states, which occur at higher fluxes and which describe the quasiholes. As we cannot add unit fluxes to a ground state with a set aspect ratio and still keep the translational symmetry intact, we choose to analyze quasiholes by either increasing $N_{x} \rightarrow N_{x}+1$ (or $N_{y} \rightarrow N_{y}+1$ ) or by removing particles from a ground state and keeping the aspect ratio fixed-hence our system sizes contain at least 3 fluxes above the ground state. A generic example is that shown in Fig. 6, in which $N=9$ particles reside in $N_{x} \times N_{y}=$ $5 \times 6$ orbitals, 3 fluxes more than the ground state at filling $1 / 3$. We diagonalize the interacting Hamiltonian for this number of particles and observe (Fig. 6) that the spectrum splits into two parts separated by a clearly visible and unambiguous energy gap. The counting of states below the gap equals the counting of quasiholes of a $1 / 3$ fractional quantum Hall state of 9 particles in 30 orbitals (explained in the next section). Even more, the counting of states per momentum sector also matches that of the generalized Pauli principle for the quasihole states [16] previously obtained in the FQH context. This will also be explained in the next section.

While for $N=9, N_{x} \times N_{y}=5 \times 6$ the gap that separates the quasihole manifold from the spurious states is

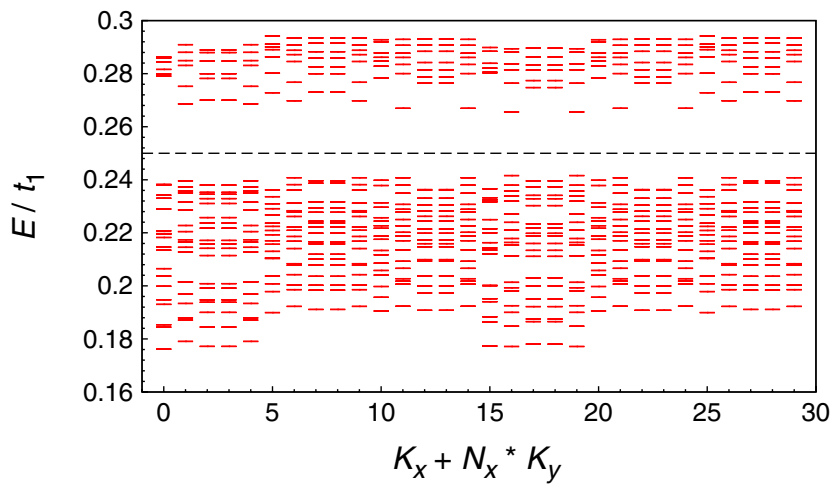

FIG. 6. Low-energy spectrum for $N=9, N_{x}=5, N_{y}=6$. The number of states below the dashed line is 19 in sectors where $K_{y} \bmod 3=0,18$ in other sectors, as expected from the analytical results. The total counting matches the one expected for Laughlin quasihole states. The counting for each momentum sector is given by the generalized Pauli principle and the 2-dimensional to 1-dimensional unfolding presented in the next section. 


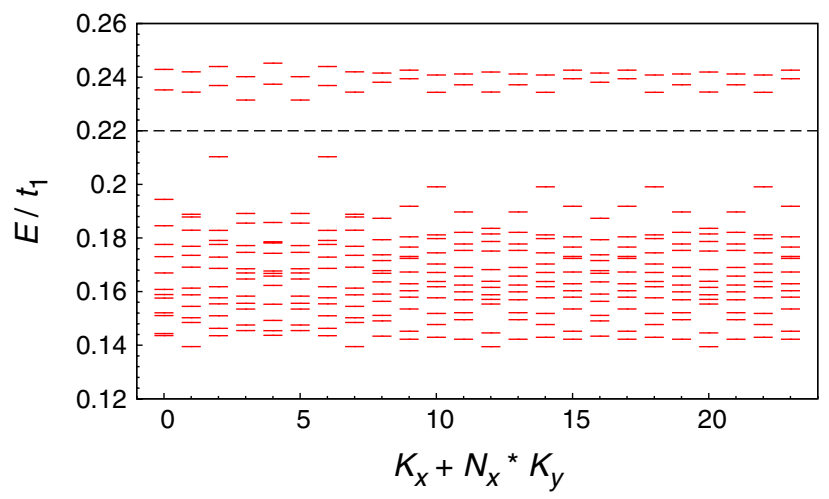

FIG. 7. Low-energy spectrum for $N=7, N_{x}=8, N_{y}=3$. The number of states below the dashed line is 12 in all sectors and matches the number of expected quasihole states given by the generalized Pauli principle.

clear, this is not always the case. In Fig. 7 we plot the spectrum of the $N=7, N_{x} \times N_{y}=8 \times 3$ problem: For most momentum sectors we find a clear gap, visible by eye, between a low-energy quasihole manifold and that of spurious, high-energy states. However, for the momenta $\left(K_{x}, K_{y}\right)=(2,0)$ [and for its inversion symmetric $(6,0)$ ] we see one state (at energy around 0.21) that lies in the gap. It is a priori unclear whether, just based on the numerical information, this state belongs to the quasihole manifold or not (the analytic counting obtained in the next section would indeed tell us that it does belong to the quasihole manifold). To find this out, we look at the level spectral flow of the states at $\left(K_{x}, K_{y}\right)=(2,0)$ upon flux insertion $k_{x} \rightarrow k_{x}+\Phi / N_{x}$ in Fig. 8, where we insert flux in the $x$ direction. We note that the state at energy 0.21 exhibits level spectral flow with the states below it, and hence it belongs to the quasihole subspace. Note that the first two excited states (around energy 0.24) do not mix with the quasihole manifold upon the flux insertion and even exhibit level repulsion between themselves. For example, when

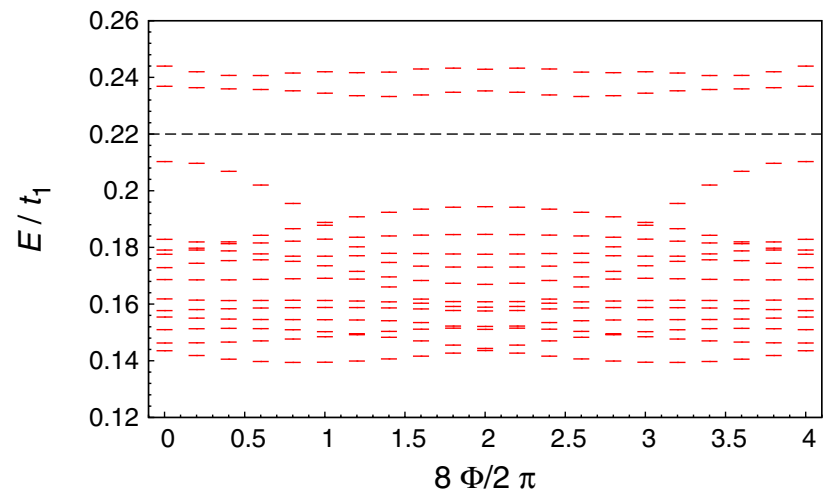

FIG. 8. Evolution of the low-energy spectrum for $N=7$, $N_{x}=8, N_{y}=3$ at $K_{x}=2, K_{y}=0$ upon flux insertion along the $x$ direction. The number of states below the dashed line is 12 for any value of $\Phi$ and matches the number of expected quasihole states given by the generalized Pauli principle
$\Phi=\pi / 8$ the lower-energy manifold is clearly separated from the spurious, high-energy one. The flux insertion tells us that the state which, in the periodic-boundary-condition case was at energy 0.21 , indeed belongs to the quasihole manifold.

A similar analysis has been made on several other system sizes and aspect ratios for quasiholes close to the $1 / 3$ filling. In the absence of a pseudopotential Hamiltonian and since we work on a lattice, several factors must be taken into account when looking at quasiholes. First, as we are diagonalizing a generic Hubbard-type Hamiltonian, we must make sure that we are still close to filling $1 / 3$. Taking out too many particles (creating too many quasiholes) could potentially lead us to another filling factor and diminish or even erase the gap between the low-energy quasiholes and the spurious high-energy states. Since we work on the lattice, care should be taken that the singleparticle system is not 1 dimensional-the aspect ratio should be roughly balanced. This will be discussed later. In most cases when these two conditions are satisfied, the gap between the quasihole manifold and the upper spurious states is visible. The total number of states (in all momentum sectors) below the spectral gap matches exactly the number of states of the Laughlin quasiholes in the regular $\mathrm{FQH}$ effect on the torus of $N$ particles and $N_{x} \cdot N_{y}$ number of orbitals. This is the number of (1,3)-admissible partitions on the torus (as defined below, see Figs. 9 and 10) that we can write down out of $N$ particles in $N_{x} \cdot N_{y}$ orbitals. For $N=8$ and $N_{x}, N_{y}=5,6$, respectively, we obtain a total of 6435 states while for $N=9$ we obtain 550 states by counting these partitions. This number is identical to the numerical data (see Figs. 6 and 7). We have checked a series of sizes up to $N=10$ and have confirmed in each case the existence of a large gap between a high-energy nonuniversal sector and a low-energy quasihole sector whose counting matches exactly that of the Laughlin quasiholes obtained by counting $(1,3)$-admissible partitions. All these quasihole states exhibit spectral flow within themselves. This proves that the observed state is an Abelian fractional quantum Hall state with $\sigma_{x y}=1 / 3$, as it has excitations obeying Haldane $1 / 3$ statistics and having the counting of a $U(1)$ boson. The overall quasihole counting matches the $(1,3)$ generalized Pauli principle. But in most cases, this statement is also valid for the counting per each momentum sector. The $(1,3)$ generalized Pauli principle is generic of the Laughlin-FQH state at filling $1 / 3$ and will be presented below.

\section{HEURISTIC COUNTING RULE: PAULI PRINCIPLE AND CRYSTAL MOMENTA}

We now present a counting rule for the total lattice momentum at which the degenerate ground states occur. A more detailed version will be developed in an upcoming paper [17]. The 2-dimensional lattice of sites $N_{x} \times N_{y}$ with $k_{x} \in\left[0, N_{x}-1\right]$ and $k_{y} \in\left[0, N_{y}-1\right]$ can be folded into a 


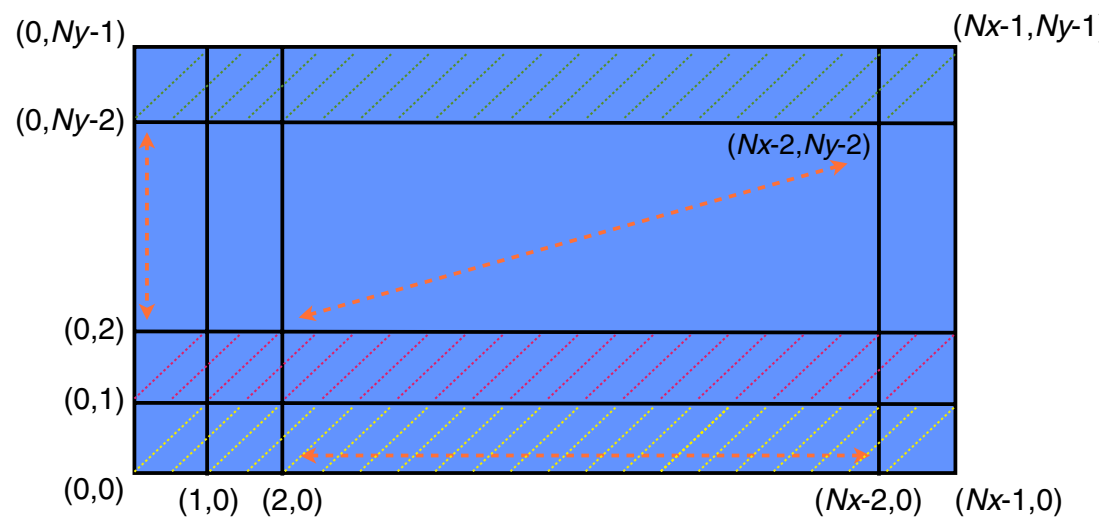

\begin{tabular}{|c|c|c|c|c|c|c|c|c|c|c|c|c|c|c|c|c|c|c|c|}
\hline$K y$ & \multicolumn{6}{|c|}{0} & \multirow{2}{*}{\multicolumn{2}{|c|}{0}} & \multirow[b]{2}{*}{2} & \multirow[b]{2}{*}{3} & \multirow{2}{*}{\multicolumn{2}{|c|}{$N_{x-1}$}} & \multirow{2}{*}{$\begin{array}{l}\ldots \\
\ldots\end{array}$} & \multicolumn{6}{|c|}{ Ny 1} \\
\hline$K x$ & 0 & 1 & 2 & 3 & 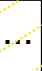 & $N_{x-}$ & & & & & & & & 0 & 1 & 2 & 3 & & $1 x$ \\
\hline $\begin{array}{l}\text { Ground } \\
\text { State } 1\end{array}$ & 1 & 0 & 0 & 1 & 0 & 0 & 1 & 0 & 0 & 1 & 0 & 0 & & 1 & 0 & 0 & 1 & 0 & 0 \\
\hline $\begin{array}{l}\text { Ground } \\
\text { State II }\end{array}$ & 0 & 1 & 0 & 0 & 1 & 0 & 0 & 1 & 0 & 0 & 1 & 0 & & 0 & 1 & 0 & 0 & 1 & 0 \\
\hline $\begin{array}{l}\text { Ground } \\
\text { State III }\end{array}$ & 0 & 0 & 1 & 0 & 0 & 1 & 0 & 0 & 1 & 0 & 0 & 1 & & 0 & 0 & 1 & 0 & 0 & 1 \\
\hline
\end{tabular}

FIG. 9. Momentum of the 3-fold degenerate ground state for the fractional Chern insulator at filling $\nu=1 / 3$. The 2-dimensional lattice of momenta $k_{x}, k_{y}$ with $k_{x} \in\left[0, N_{x}-1\right]$ and $k_{y} \in\left[0, N_{y}-1\right]$ (in units of $2 \pi / N_{x}, 2 \pi / N_{y}$, respectively) where we have taken $N_{x}$ to be a multiple of 3 (since $N_{x} \cdot N_{y}=3 N$, this can always be done) is unfolded in a 1D lattice. The total momentum of the ground states is then the same as the momentum of the three (1,3)-admissible partitions possible for the number of orbitals $N_{x} \cdot N_{y}$ and number of particles $N=N_{x} \cdot N_{y} / 3$.

1-dimensional lattice of orbitals of momentum $\lambda=$ $N_{x} k_{y}+k_{x}$ by placing the $x$-momentum range one after another for the different values of the $y$ momenta, as shown in Fig. 9. This then represents a map between the momentum of the $i$-th particle orbital $k_{x, i}, k_{y, i}$ and the 1-dimensional momentum of the $i$-th orbital $\lambda_{i} \in$ $\left[0, N_{x} \cdot N_{y}-1\right]$. The $\lambda_{i}$ 's then form a partition $\lambda=$ $\left[\lambda_{1}, \ldots, \lambda_{N}\right]$, which we order from highest to lowest.

\begin{tabular}{|c|c|c|c|c|c|c|c|c|c|c|c|c|c|c|}
\hline$k_{y}$ & \multicolumn{3}{|c|}{0} & \multicolumn{3}{|c|}{1} & \multicolumn{3}{|c|}{2} & \multicolumn{3}{|c|}{3} & \multicolumn{2}{|c|}{ Total Momentum } \\
\hline$k x$ & 0 & 1 & 2 & 0 & 1 & 2 & 0 & 1 & 2 & 0 & 1 & 2 & $K_{x}$ & $K_{y}$ \\
\hline Ground State 1 & 1 & 0 & 0 & 1 & 0 & 0 & 1 & 0 & 0 & 1 & 0 & 0 & $\begin{array}{c}\begin{array}{c}0+0+0+0 \\
=0(\bmod 3)=0\end{array} \\
\end{array}$ & $\begin{array}{c}\begin{array}{c}0+1+2+3 \\
=6(\bmod 4)=2\end{array}\end{array}$ \\
\hline Ground State II & 0 & 1 & 0 & 0 & 1 & 0 & 0 & 1 & 0 & 0 & 1 & 0 & $\begin{aligned} & 1+1+1+1 \\
&=(\bmod 3)=1 \\
&\end{aligned}$ & $\begin{array}{c}0+1+2+3 \\
=6(\bmod 4)=2 \\
=\end{array}$ \\
\hline Ground State III & 0 & 0 & 1 & 0 & 0 & 1 & 0 & 0 & 1 & 0 & 0 & 1 & $\begin{array}{c}2+2+2+2 \\
=8(\bmod 3)=2\end{array}$ & $\begin{array}{c}0+1+2 \\
=6(\bmod 4)=2\end{array}$ \\
\hline
\end{tabular}

\begin{tabular}{|c|c|c|c|c|c|c|c|c|c|c|c|c|c|c|}
\hline$k_{y}$ & \multicolumn{4}{|c|}{0} & \multicolumn{4}{|c|}{1} & \multicolumn{4}{|c|}{2} & \multicolumn{2}{|c|}{ Total Momentum } \\
\hline$k x$ & \begin{tabular}{|l|l}
0 & 1
\end{tabular} & 23 & 4] & & 1 & 2 & $\begin{array}{lll}3 & 4\end{array}$ & 5 & 01 & 12 & & 5 & $K_{x}$ & $K_{y}$ \\
\hline Ground State 1 & \begin{tabular}{|l|l|}
1 & 0 \\
\end{tabular} & $\begin{array}{lll}0 & 1 \\
\end{array}$ & . & 이 & 10 & 0 & 10 & 0 & 1 & 0 & & 0 & $\begin{array}{l}0+3+0+3+0+3 \\
=9(\bmod 6)=3\end{array}$ & $\begin{array}{l}0+0+1+1+2+2 \\
=6(\bmod 3)=0\end{array}$ \\
\hline Ground State II & \begin{tabular}{l|l|l}
0 & 1 &
\end{tabular} & 0 & 1 & 이 & 1 & 0 & $\begin{array}{ll}0 & 1\end{array}$ & 0 & & 0 & & 0 & $\begin{array}{l}1+4+1+4+1+4 \\
=15(\bmod 6)=3\end{array}$ & $\begin{array}{l}0+0+1+1+2+2 \\
=6(\bmod 3)=0\end{array}$ \\
\hline Ground State III & $\begin{array}{lll}0 & 0\end{array}$ & 10 & 0 & 1 [ & 0 & 1 & 00 & 1 & 010 & 1 & 00 & 1 & $\begin{array}{l}2+5+2+5+2+5 \\
=21(\bmod 6)=3\end{array}$ & $\begin{array}{l}0+0+1+1+2+2 \\
=6(\bmod 3)=0\end{array}$ \\
\hline
\end{tabular}

FIG. 10. Example of the total momentum counting for the 3-fold degenerate ground states of the $N=4$ (top) and $N=6$ (bottom) problem. Every time the number of particles $N$ is a multiple of both $k_{x}, k_{y}$, the 3 -fold degenerate ground states occur at the same total momentum and are expected to be split by the interaction for finite-size samples. Notice that for the $N=4$ problem, the total momenta at which the ground state occurs are related by inversion symmetry, as $(1,2)=(-2,-2) \bmod (3,4)$. 


\begin{tabular}{|c|c|c|c|c|c|c|c|c|c|c|c|}
\hline$k y$ & \multicolumn{3}{|c|}{0} & \multicolumn{3}{|c|}{1} & \multicolumn{3}{|c|}{2} & \multicolumn{2}{|c|}{ Total Momentum } \\
\hline$k x$ & 0 & 1 & 2 & 0 & 1 & 2 & 0 & 1 & 2 & $K x(\bmod 3)$ & $K y(\bmod 3)$ \\
\hline \multirow{2}{*}{$\begin{array}{l}\text { Two states at total } \\
\text { momentum }(0,0)\end{array}$} & 0 & 0 & 0 & 1 & 0 & 0 & 1 & 0 & 0 & 0 & 0 \\
\hline & 0 & 0 & 0 & 0 & 1 & 0 & 0 & 0 & 1 & 0 & 0 \\
\hline \multirow{2}{*}{$\begin{array}{l}\text { Two states at total } \\
\text { momentum }(1,0)\end{array}$} & 0 & 0 & 0 & 1 & 0 & 0 & 0 & 1 & 0 & 1 & 0 \\
\hline & 0 & 0 & 0 & 0 & 0 & 1 & 0 & 0 & 1 & 1 & 0 \\
\hline \multirow{2}{*}{$\begin{array}{l}\text { Two states at total } \\
\text { momentum }(2,0)\end{array}$} & 0 & 0 & 0 & 1 & 0 & 0 & 0 & 0 & 1 & 2 & 0 \\
\hline & 0 & 0 & 0 & 0 & 1 & 0 & 0 & 1 & 0 & 2 & 0 \\
\hline \multirow{2}{*}{$\begin{array}{l}\text { Two states at total } \\
\text { momentum }(0,1)\end{array}$} & 1 & 0 & 0 & 1 & 0 & 0 & 0 & 0 & 0 & 0 & 1 \\
\hline & 0 & 1 & 0 & 0 & 0 & 1 & 0 & 0 & 0 & 0 & 1 \\
\hline \multirow{2}{*}{$\begin{array}{l}\text { Two states at total } \\
\text { momentum }(1,1)\end{array}$} & 1 & 0 & 0 & 0 & 1 & 0 & 0 & 0 & 0 & 1 & 1 \\
\hline & 0 & 0 & 1 & 0 & 0 & 1 & 0 & 0 & 0 & 1 & 1 \\
\hline \multirow{2}{*}{$\begin{array}{l}\text { Two states at total } \\
\text { momentum }(2,1)\end{array}$} & 1 & 0 & 0 & 0 & 0 & 1 & 0 & 0 & 0 & 2 & 1 \\
\hline & 0 & 1 & 0 & 0 & 1 & 0 & 0 & 0 & 0 & 2 & 1 \\
\hline \multirow{2}{*}{$\begin{array}{l}\text { Two states at total } \\
\text { momentum }(0,2)\end{array}$} & 1 & 0 & 0 & 0 & 0 & 0 & 1 & 0 & 0 & 0 & 2 \\
\hline & 0 & 0 & 1 & 0 & 0 & 0 & 0 & 1 & 0 & 0 & 2 \\
\hline \multirow{2}{*}{$\begin{array}{l}\text { Two states at total } \\
\text { momentum }(1,2)\end{array}$} & 0 & 1 & 0 & 0 & 0 & 0 & 1 & 0 & 0 & 1 & 2 \\
\hline & 0 & 0 & 1 & 0 & 0 & 0 & 0 & 0 & 1 & 1 & 2 \\
\hline \multirow{2}{*}{$\begin{array}{l}\text { Two states at total } \\
\text { momentum }(2,2)\end{array}$} & 0 & 1 & 0 & 0 & 0 & 0 & 0 & 1 & 0 & 2 & 2 \\
\hline & 0 & 0 & 1 & 0 & 0 & 0 & 1 & 0 & 0 & 2 & 2 \\
\hline
\end{tabular}

FIG. 11. Example of total momentum counting for the quasiholes of the $N=2, N_{x}=N_{y}=3$ problem.

This partition is then similar to the orbital momenta of the particles of the usual FQH on the torus. On such geometry and in the lowest Landau level, the singleparticle orbitals can be written as

$$
\begin{aligned}
\phi_{\lambda}^{\mathrm{FQH}}(x, y)= & \sum_{m \in \mathbb{Z}} e^{2 \pi / L_{y}\left(\lambda+m N_{\phi}\right)(x+i y)} e^{-\left(x^{2} / 2 l_{b}^{2}\right)} \\
& \times e^{-(1 / 2)\left(2 \pi l_{b} / L_{y}\right)^{2}\left(\lambda+m N_{\phi}\right)^{2}} .
\end{aligned}
$$

In the above, we have picked the Landau gauge $\vec{A}=B x \vec{e}_{y}$. $l_{b}$ is the magnetic length, $N_{\phi}$ is the number of flux quanta, and $L_{y}$ is the system size along $y$. With this gauge choice, $\lambda$ is the orbital momentum along the $y$ direction and is such that $\lambda \in\left[0, N_{\phi}-1\right]$. Setting $N_{\phi}=N_{x} \cdot N_{y}$, we can formally relate the FQH momentum $\lambda$ and the linearized momentum of the fractional Chern insulator $\lambda=N_{x} k_{y}+k_{x}$.

Using this analogy between $\mathrm{FQH}$ on the torus and fractional Chern insulator, we now index each many-body state by a single partition (called "root" partition [16]) that satisfies a generalized Pauli principle (also called an "admissibility condition"), which does not allow the existence of more than 1 particle in 3 consecutive orbitals. In partition notation, this reads $\lambda_{i}-\lambda_{i+1} \geq 3$. Because of the periodicity of the torus, we must also make sure that the first and last particles are separated by at least 3 orbitals, which reads $\lambda_{N}+N_{x} \cdot N_{y}-1-\lambda_{1} \geq 3$. At the ground-state filling $N=N_{x} \cdot N_{y} / 3$ (we pick $N_{x}$ a multiple of 3 ) there are only three such partitions that correspond to the occupation numbers 100100100...100100 $\left[K_{x}, K_{y}=N_{x}\left(N_{x}-3\right) / 6, N\left(N_{y}-1\right) / 2 \bmod \left(N_{x}, N_{y}\right)\right]$, 010010010. .010010 $\left[K_{x}, K_{y}=N_{x}\left(N_{x}-3\right) / 6+N\right.$, $\left.N\left(N_{y}-1\right) / 2 \bmod \left(N_{x}, N_{y}\right)\right]$, and $00100100100 \ldots 001001$ $\left[K_{x}, K_{y}=N_{x}\left(N_{x}-3\right) / 6+2 N, N\left(N_{y}-1\right) / 2 \bmod \left(N_{x}, N_{y}\right)\right]$, of the orbitals of momentum $\lambda_{1}, \lambda_{2}, \ldots, \lambda_{N}$. As an empirical observation, it was noted in [7] that the degenerate ground states appear at momenta related by a translation with the same number in both $x, y$ directions. This is an immediate corollary of the counting principle presented above. In the present model, due to inversion symmetry, at least two of the ground states are exactly degenerate. The principle for finding the ground-state momentum reminds one of the root partitions used in the usual FQH effect [16] or in the thin-torus limit of the FQH states [18].

At this point, a discussion of the dependence of the physics on the aspect ratio of the problem is necessary. In cases of finite-size systems, the exact-diagonalization data for the momentum of the 3 quasidegenerate ground states matches the momentum counting presented above for aspect ratios for which the system is 2 dimensional. The Chern-insulator problem has the particular property that the filled band only has a Chern number (Hall conductance) equal to unity in the case of $N_{x} / N_{y} \rightarrow$ finite. For example, if we let $N_{y}=1$ and $N_{x} \rightarrow \infty$, the Chern number of this Hamiltonian will not be equal to unity. The Chern number is equal to $\int d k_{x} d k_{y} \operatorname{Tr}\left(P\left[\partial_{k_{x}} P, \partial_{k_{y}} P\right]\right) /(2 \pi)^{2}$ and hence $N_{y}$ cannot take small values such as 1,2 in order for the derivatives of the Chern number to be well defined. The Brillouin zone mesh of momenta must truly be 
2 dimensional in order for the filled band to have an integer Chern number. We hence expect that the interacting problem also be sensitive to the aspect ratio when one of the dimensions becomes much smaller than the other (of course in the thermodynamic limit, as long as $N_{x} / N_{y}$ remains finite, we expect the degeneracy of the $\mathrm{FQH}$ states to be independent of the aspect ratio). In fact, the interacting problem has also a different dependence on the aspect ratio. To see this, one can look at the extreme case of $N_{y}=1, N_{x}=3 N$. In this case, we are indeed solving a 1-dimensional problem of orbitals $k_{x}=0, \ldots, 3 N-1-$ extremely similar to that of the LLL orbitals on the torus-but with interacting Hamiltonian that comes from a 2D Hubbard interaction. This Hamiltonian is much different from the Haldane pseudopotential Hamiltonian which involves Clebsch-Gordon coefficients on the sphere and Jacobi theta functions on the torus. As such, the problem is physically distinct at the interacting level, and for these skewed aspect ratios we do not expect $\mathrm{FQH}$ states in the Chern-insulator problem. Indeed, in the finite-size diagonalizations performed below, we will always aim to choose the most symmetric aspect ratio available. As observed in Fig.s 3 and 4, the cleanness of the results will depend on this choice, as it is natural for these finite sizes.

The counting of quasiholes per momentum sector is slightly more complicated, and we have not found the full counting rule per momentum sector. The map between the 2-dimensional momentum numbers and the 1-dimensional orbital partition that allowed us to find the momentum of the ground states cannot always work for the quasihole states. It sometimes violates the inversion symmetry (see the Appendix for such cases) and thus it sometimes does not match the numerical results. Nevertheless, the total number of quasiholes, irrespective of the momentum sectors, always matches the counting of partitions. This matching of the exact diagonalization states and the 1.3-admissible partitions is a rather remarkable demonstration of the $\nu=1 / 3$ character of the states. Even more interestingly, the Pauli principle and the map between the 2-dimensional momentum numbers and the 1-dimensional orbital partition works for each momentum sector (not only for the total number of quasiholes) in the great majority of the cases studied. As an example, we present in Fig. 11 the generalized Pauli counting principle at work for $N=2, N_{x}=N_{y}=3$.

\section{ENTANGLEMENT SPECTRUM}

We now turn back to the 3-fold degenerate ground state of the system. There are several attempts to obtain an analytical expression of the Laughlin state for the fractional Chern insulators [19,20]. Unfortunately, none of them can be used in our case, so we cannot perform any wavefunction overlap calculations to compare their analytical expression with the ground state of our system.
Nevertheless, we can still show that the ground state contains, by itself, information about the Abelian fractional $1 / 3$ character of the excitation spectrum. This is actually a far better probe than an overlap, since the $1 / 3$ character is the feature, not the analytical expression. To do this, we use the recently developed entanglement spectrum [21,22], which for a single nondegenerated ground state $|\Psi\rangle$ can be defined through the Schmidt decomposition of $|\Psi\rangle$ in two regions $A, B$ (not necessarily spatial):

$$
|\Psi\rangle=\sum_{i} e^{-\xi_{i} / 2}\left|\Psi_{i}^{A}\right\rangle \otimes\left|\Psi_{i}^{B}\right\rangle,
$$

where $\left\langle\Psi_{i}^{A} \mid \Psi_{j}^{A}\right\rangle=\left\langle\Psi_{i}^{B} \mid \Psi_{j}^{B}\right\rangle=\delta_{i, j}$. The $\exp \left(-\xi_{i}\right)$ and $\left|\Psi_{i}^{A}\right\rangle$ are the eigenvalues and eigenstates of the reduced density matrix, $\rho_{A}=\operatorname{Tr}_{B} \rho$, where $\rho=|\Psi\rangle\langle\Psi|$ is the total density matrix. There is no generalization of (5) to degenerate ground states. Still the definition of the entanglement spectrum through the reduced density matrix can be extended to that case. While several schemes can be proposed, it has been observed in [22] that the incoherent summation over the degenerate ground states $\rho=$ $\frac{1}{3} \sum_{i}\left|\Psi_{i}\right\rangle\left\langle\Psi_{i}\right|$ is a good candidate for this generalization. This combination builds a density matrix which commutes with the total translation operators, which is a desired feature to sort the $\xi_{i}$ with respect to the momentum quantum numbers.

Depending on the space where the system is split into $A$ and $B$, be it real, momentum or particle space, different aspects of the system excitations will be revealed through the ES. It was proven that if the regions $A, B$ are regions of particles [22], the particle entanglement spectrum hence obtained by tracing over the positions of a set of $B$ particles gives information about the number of quasiholes of the system of $N_{A}$ particles and the number of orbitals identical to that of the untraced system. In the case of the usual $\mathrm{FQH}$, the particle entanglement spectrum of a model state contains an identical number of levels (i.e., the number of non zero eigenvalues) as those of the quasiholes. Thus, the counting of nonzero eigenvalues does not suffer from the sometimes uncontrolled finite-size effects, as observed with state decomposition in the momentum space $[21,23]$. For this reason we will use state decomposition in the particle space as a probe for the fractional Chern insulator.

Away from the model states, like the Coulomb ground state, the ES may exhibits an entanglement gap [21,24]. It separates a low-energy structure with perfect quasihole counting and a high-entanglement-energy nonuniversal part. But a clear and significant gap is not always observed, even for the $\nu=1 / 3$ Coulomb state.

For the fractional Chern insulator, the situation is surprisingly much better: We observe a clear, large entanglement gap between low-entanglement-energy levels and the high-entanglement-energy levels like those observed in Figs. 12 and 13. Moreover, the counting of the levels below the gap is identical to the counting of quasiholes of $N_{A}$ 


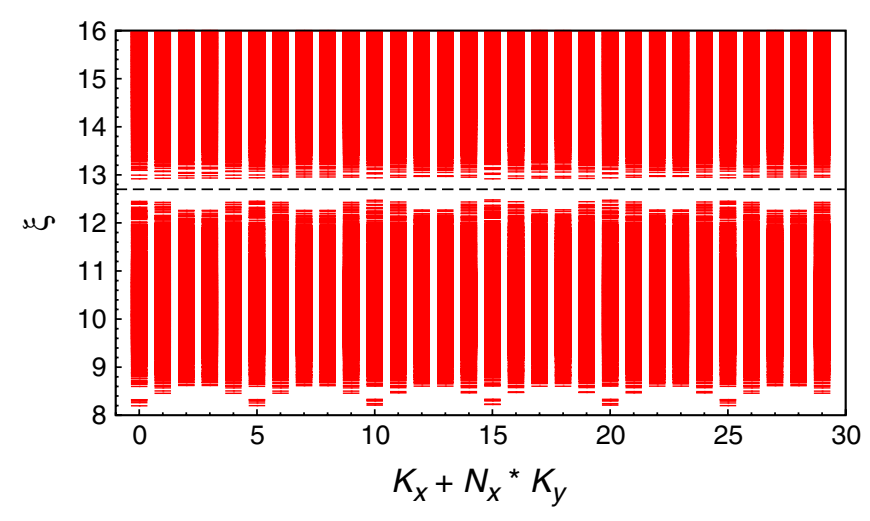

FIG. 12. Particle entanglement spectrum for $N=10, N_{x}=5$, $N_{y}=6$, and $N_{A}=5$. The $\xi$ 's are the entanglement energies. The number of states below the dashed line is 776 in all the $k_{x}=0$, and 775 in the other sectors. This is in agreement with the $(1,3)$ Pauli principle for every momentum sector.

particles in $N_{x} \cdot N_{y}$ orbitals. In both examples shown in Figs. 12 and 13, it matches per each momentum sector that of $(1,3)$-admissible partitions of $N_{A}$ particles in $N_{x} \cdot N_{y}$ orbitals. We find it very revealing that the fractional-Cherninsulator ground states obtained here contain much clearer information (large, clear entanglement gap) than the ground states of the Coulomb interaction in the FQH. The entanglement spectrum shows that the ground states by themselves contain essential information on the fractional nature of the excitations in the fractional Chern insulator. The current clean application of the entanglement spectrum also shows that this quantity is fundamentally useful toward revealing the physics of strongly-correlated states besides the usual FQH model wave functions for $\mathrm{FQH}$ states.

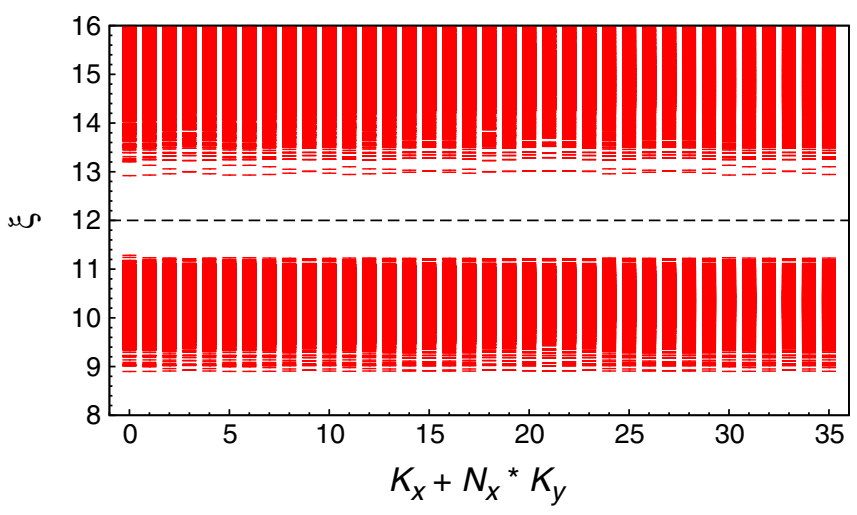

FIG. 13. Particle entanglement spectrum for $N=12, N_{x}=$ $N_{y}=6$, and $N_{A}=4$. The $\xi$ 's are the entanglement energies. The number of states below the dashed line is 741 in momentum sectors where $N_{x} \bmod 2=N_{y} \bmod 2=0$ and 728 elsewhere. The total number below this line (26325) exactly matches the one predicted by the counting rule.
The ES also provides some insight about the system when the number of sites in one direction is equal to 3 . A clear entanglement gap is observed and the counting below this gap matches the expected one, but only for $N_{A}=2$ and $N_{A}=3$. For larger values of $N_{A}$, the number of levels below the gap is lower than expected. This strongly suggest that for the small aspect ratios where the ratio $N_{y} / N_{x} \rightarrow 0$, the state is not a fully developed fractional Chern insulator-or indeed, by our previous arguments, the single-particle problem is not even a welldeveloped integer Chern insulator. In the case of the other aspect ratios, all the ESs for any value of $N_{A}$ and any system sizes up to $N=12$ perfectly matche the predicted counting. For the cases such as $N_{x} \times N_{y}=6 \times 5$ and $6 \times 6$, the $(1,3)$ Pauli-principle counting matches the ES data for each momentum sector for all $N_{A}=1,2,3,4,5$. The perfect match clearly shows that this state is a fractional Chern insulator.

\section{TRANSITION TO THE TRIVIAL INSULATOR}

Tuning the mass term above the $4 t_{2}$ threshold yields an insulator topologically equivalent to the atomic limit, with zero Hall conductance. Partially filling this insulator at say $M=6 t_{2}$, we find no clear sign of a 3 -fold degenerate ground state, and no clear gap to the excitation spectrum (see Fig. 14). In the interacting problem, we would think that the gap rearrangement happens more quickly than $M / 4 t_{2}$. This hypothesis is natural since the many-body gap is expected to be more sensitive than the single-particle one. The evolution of the many-body gap with the parameter $M$ is plotted in Fig. 15. In the atomic limit $M \rightarrow \infty, B$ sites have an energy $-M$ and all the particles are strongly localized on those sites. If all $B$ sites were occupied and $A$ sites unoccupied, the filling would be $1 / 2$, and hence as we are at a smaller filling $1 / 3$, and as the interaction couples sites $A$ and $B$, the ground state will be highly degenerate. We see that the many-body gap as a function of $M$ collapses at exactly the value of $M$ at which the one-body transition takes place. We also plot the entanglement gap $\Delta_{\text {PES }}$ as a function of $M$ in Fig. 16. The entanglement gap is

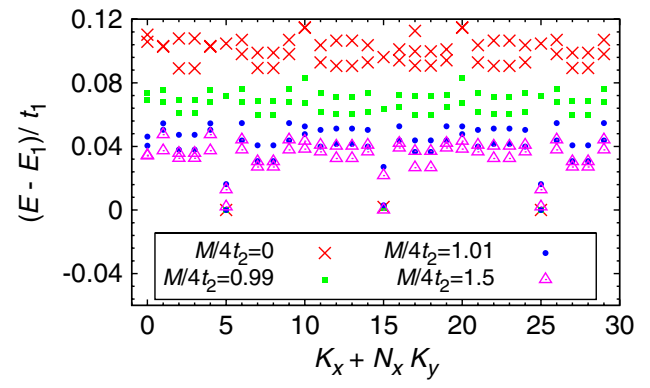

FIG. 14. Low-energy spectrum for different values of $M$ at $N=10, N_{x}=5$, and $N_{y}=6$. We show only the two lowest energies per momentum sector. All energies are shifted such that the ground-state energy is 0 for each $M$ value. 


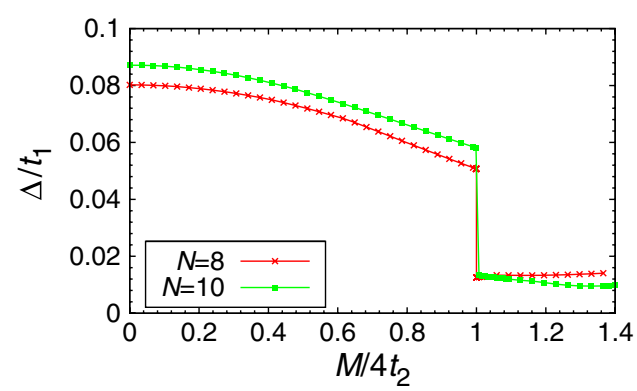

FIG. 15. Energy gap $\Delta$ as a function of $M$ at filling $\nu=1 / 3$ for $N=8$ and $N=10$ with $N_{x}=N / 2, N_{y}=6$. For both system sizes, the transition occurs at $M=4 t_{2}$.

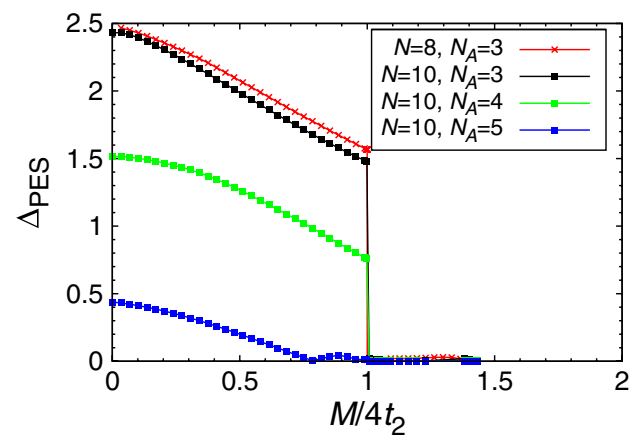

FIG. 16. Entanglement spectrum gap $\Delta_{\text {PES }}$ as a function of $M$ at filling $\nu=1 / 3$ for $N=8$ and $N=10$ with $N_{x}=$ $N / 2, N_{y}=6$.

defined as the minimum separation, over the different momenta sectors, between the higher entanglement energy level belonging to the Laughlin counting and the lower entanglement energy level that does not belong to this counting. $\Delta_{\text {PES }}$ tracks the energy gap and its collapse also at the same value as the many-body and single-particle energy gaps.

\section{ANALYTICAL COUNTING OF QUASIHOLES PER MOMENTUM SECTOR}

The heuristic generalized Pauli-principle counting of the previous section gives the correct (matching the data) total counting of quasihole-state manifold at any $N, N_{y}, N_{x}$ (as long as the aspect ratio is 2 dimensional). In most cases, although not in all, it also gives the correct counting per each momentum sector. The generalized Pauli principle is based on the correspondence between a 2D momenta map and 1D orbitals, which is heuristic. In this section, we provide some analytic results of the counting per momentum sector that has been tested to work for all the momentum sectors and is not based on a 2D-to-1D folding. The counting of the total number of quasihole states of a $1 / 3$ state of $N$ particles in $N_{x} \cdot N_{y}$ orbitals on the torus can be obtained by counting the number of ways of putting $N$ particles in $N_{x} \cdot N_{y}$ orbitals with the restriction that there should be no more than 2 particles in 3 consecutive orbitals [(1,3)-admissibl- partitions-generalized Pauli principle] on the sphere:

$$
\#_{\text {qhsphere }}=\left(\begin{array}{c}
N+n \\
n
\end{array}\right), \quad n=N_{x} N_{y}+2-3 N,
$$

where $n$ is the number of quasiholes, and then subtracting the configurations that violate the $(1,3)$ generalized Pauli principle once we make the system periodic to go to the torus. These configurations come in only three possible ways, and can be written in terms of the occupation number of orbitals

$$
\begin{aligned}
& 0100[\text { all }(1,3) \text {-admissible configurations }] 001, \\
& 100[\text { all }(1,3) \text {-admissible configurations }] 0010, \\
& 100[\text { all }(1,3) \text {-admissible configurations }] 001 .
\end{aligned}
$$

These represent the only sets of configurations that are allowed on the sphere but that have more than one particle in 3 consecutive orbitals if the orbital space is made periodic. The number of the first two are equal by inversion symmetry and reads as

$$
\left(\begin{array}{c}
N-2+n^{\prime} \\
n^{\prime}
\end{array}\right), \quad n^{\prime}=N_{x} N_{y}+1-3 N
$$

while the third one reads as

$$
\left(\begin{array}{c}
N-2+n^{\prime \prime} \\
n^{\prime \prime}
\end{array}\right), \quad n^{\prime \prime}=N_{x} N_{y}+2-3 N .
$$

The result then reads as

$$
\#_{\mathrm{qh} \mathrm{torus}}=N_{x} N_{y} \frac{\left(N_{x} N_{y}-2 N-1\right) !}{N !\left(N_{x} N_{y}-3 N\right) !} .
$$

This is the total number of quasiholes of a $1 / 3$ state on the torus in a translationally invariant system, and for a an incompressible and featureless $\mathrm{FQH}$ topological state, there is no particular reason why some momenta should have an occupation number different from others. Indeed, both the interacting-particle and the single-particle models are translationally invariant, and as such in the featureless liquid ground state, momenta occupation numbers should be the same. Hence, on a finite-size lattice for which we have $N_{x} \cdot N_{y}$ momenta, we would expect to have $\frac{\left(N_{x} N_{y}-2 N-1\right) !}{N !\left(N_{x} N_{y}-3 N\right) !}$ states for each momenta. While this is always certainly true in the thermodynamic limit (as in the 
heuristic Pauli principle), there are cases of finite-size systems when $\frac{\left(N_{x} N_{y}-2 N-1\right) !}{N !\left(N_{x} N_{y}-3 N\right) !}$ is not an integer. This means that in finite-size cases, commensuration effects weakly modify the finite-size counting of each sector so that the requirement that there be an integer number of quasihole states for each momentum sector is satisfied. This is the reason for the finite-size changes of the number of states in each momentum sector.

Physical reasoning leads us further. When $N$ is relatively prime with both $N_{x}$ and $N_{y}$ there can be no commensuration effects, and the number of quasihole states per each momentum sector has to be identical. Indeed, in this case, $\frac{\left(N_{x} N_{y}-2 N-1\right) !}{N !\left(N_{x} N_{y}-3 N\right) !}$ is an integer, is equal to the number of states per momentum sector provided by our heuristic Pauli principle, and matches the numerical data for the number of states per momentum sector. This is also the asymptotic value of the number of states per momentum sector in the thermodynamic limit.

Finite-size effects become important in the case when the greatest common divisors (GCD) of $N, N_{x}$ or $N, N_{y}$ is larger than 1. Three scenarios can occur. First, $\operatorname{GCD}\left(N, N_{y}\right)>1, \operatorname{GCD}\left(N, N_{x}\right)=1$ : In this case, the number of quasihole states is the same for all the $K_{y}$ total momenta that are divisible by $\operatorname{GCD}\left(N, N_{y}\right)$ and is different from the number of states at $K_{y}$ total momenta not divisible by $\operatorname{GCD}\left(N, N_{y}\right)$. A clear example of this situation is in Fig. 6: $N=9, N_{x}=5, N_{y}=6$, and hence $\operatorname{GDC}\left(N, N_{y}\right)=$ 3. The momenta $\left(K_{x}, K_{y}\right)$ for which $K_{y} \bmod 3=0$ have 19 states in the quasihole subspace, whereas all momenta for which $K_{y} \bmod 3 \neq 0$ have 18 states in the quasihole subspace. This is also equivalent to the counting of $(1,3)$ partitions obeying the generalized Pauli principle. The case $\operatorname{GCD}\left(N, N_{x}\right)=1, \operatorname{GCD}\left(N, N_{x}\right)>1$ is obviously the $x \leftrightarrow y$ of the case presented above.

Second, when $\operatorname{GCD}\left(N, N_{y}\right)=\operatorname{GCD}\left(N, N_{x}\right)>1$, the number of quasihole states is the same for all $K_{x}, K_{y}$, which are simultaneously both divisible by $\operatorname{GCD}\left(N, N_{y}\right) \times$ $\left[=\operatorname{GCD}\left(N, N_{x}\right)\right]$ and different from the number of quasihole states at $K_{x}, K_{y}$ when either $K_{x}$ or $K_{y}$ is not divisible by $\operatorname{GCD}\left(N, N_{y}\right)$. An example of this latter situation is in the counting of the entanglement spectrum states of the $N=12\left(N_{x}=6, N_{y}=6\right)$ particles' ground state and $N_{A}=4$ in Fig. 13. The counting of states in the entanglement spectrum should be identical with that of $N_{A}=4$ quasiholes in $N_{x} \cdot N_{y}=36$ orbitals. As such, $\operatorname{GCD}\left(N, N_{x}\right)=\operatorname{GCD}\left(N, N_{y}\right)=2$. Momenta $\left(K_{x}, K_{y}\right)=$ $(0,0),(2,0),(4,0),(0,2),(2,2),(4,2),(0,4),(2,4),(4,4)$, which are both divisible by $\operatorname{GCD}\left(N, N_{x}\right)$ and have 741 quasihole states, whereas all other momenta, in which either $K_{x}$ or $K_{y}$ are not divisible by $\operatorname{GCD}\left(N, N_{x}\right)$, have 728 quasihole states, for a total of 26325 states, which matches the total number of states of the $(1,3)$ Pauli principle. These rules for the counting of states are physically motivated by the fact that momenta on the lattice should be filled democratically except in the case when the number of particles exhibits some commensuration [expressed by us as the condition $\operatorname{GCD}\left(N, N_{x, y}\right)>1$ ] with the lattice dimensions. They have been checked for a range of particle numbers (up to $N=12$ ) and found to hold in all cases. The rules make sense physically: In the case of commensuration, the momenta that are divisible by the commensuration factor (GCD) should all exhibit the same counting (once the commensuration condition has been established, the commensurate momenta are treated democratically). This counting should be different from the counting of the momenta incommensurate with the GCD. Whenever either $\operatorname{GCD}\left(N, N_{x}\right)$ or $\operatorname{GCD}\left(N, N_{y}\right)$ equal unity, the Pauliprinciple counting of states matches the numerically found counting.

Third, $\operatorname{GCD}\left(N, N_{y}\right) \neq \operatorname{GCD}\left(N, N_{x}\right) \neq 1$ : This case has not been analyzed and compared to the numerical data (as the size of the numerical computation becomes too large). However, we can offer a conjecture for the quasihole state counting per momentum. $\left(K_{x}, K_{y}\right)$ momenta, which are not multiples of either $\operatorname{GCD}\left(N, N_{y}\right)$ or $\operatorname{GCD}\left(N, N_{x}\right)$, have identical counting, different from all others. The momenta for which $\operatorname{GCD}\left(N, N_{x}\right)$ divides $K_{x}$ but $\operatorname{GCD}\left(N, N_{y}\right)$ does not divide $K_{y}$ all have the same counting, different from any other. This is similar for momenta for which $\operatorname{GCD}\left(N, N_{y}\right)$ divides $K_{y}$ but $\operatorname{GCD}\left(N, N_{x}\right)$ does not divide $K_{x}$. Finally, momenta for which $K_{x}$ is divisible by $\operatorname{GCD}\left(N, N_{x}\right)$ and $K_{y}$ is divisible by $\operatorname{GCD}\left(N, N_{y}\right)$ have same counting, different from the rest.

\section{CONCLUSION}

In conclusion, we have shown that the ground state of the $\nu=1 / 3$ flat-band Chern insulator in the presence of repulsive interactions is an incompressible state with Hall conductance $1 / 3$, and that quasihole excitations satisfying fractional statistics. The presence of the excitation whose counting is identical to those in the $\mathrm{FQH} \nu=1 / 3$ state is a clear and up-to-now missing proof that the ground state is indeed a fractional Chern insulator and not a 1/3 chargedensity-wave state. We have presented a mapping between the lattice momenta and the torus orbitals and shown that the counting and total momenta of the ground states and those of the quasiholes can be obtained by employing a generalized Pauli principle of not more than 2 particles in 3 consecutive orbitals. We have then shown that the entanglement spectrum of the ground state also has an entanglement gap and that the levels below the gap match in counting those of the Laughlin quasihole states.

\section{ACKNOWLEDGMENTS}

BAB wishes to thank T. L. Hughes, F. D. M. Haldane, S. Sondhi, and C. Chamon and L. Santos for very useful discussions. B. A. B. was supported by Princeton Startup 
Funds, Sloan Foundation, NSF DMR-095242, NSF China 11050110420, and a MRSEC grant at Princeton University, NSF DMR-0819860.

\section{APPENDIX: QUASIHOLES AND THE GENERALIZED PAULI PRINCIPLE}

The counting of quasiholes per momentum sector is slightly more complicated, and we have not found the full counting rule per momentum sector. Although the total number of quasiholes always matches the counting of partitions, the map between the 2-dimensional momentum numbers and the 1-dimensional orbital partition that allowed us to find the momentum of the ground states cannot always work for the quasihole states. This can be easily seen as the partition map between the momentum of the $i^{\prime}$-th particle $k_{x i}, k_{y i}$, and the 1-dimensional momentum (1,3)-admissible partition $\lambda=\left(\lambda_{1}, \ldots, \lambda_{N}\right)$ with $\lambda_{i}=$ $N_{x} \cdot k_{y i}+k_{x i}$ does not (for quasiholes) have to respect the inversion symmetry that the model studied has. To illustrate this, take two particles $i, j$, their momenta $\left(k_{x i}, k_{y i}\right),\left(k_{x j}, k_{y j}\right)$, and their orbital momentum $\lambda_{i}, \lambda_{j}$ satisfying the $(1,3)$ admissibility $\left|\lambda_{i}-\lambda_{j}\right| \geq 3$. The inversion property acts at the single-particle level and transforms $k_{x}, k_{y} \rightarrow-k_{x},-k_{y}$. To make the inverted momentum belong to the positive numbers, we must add $N_{x}, N_{y}$ to the $x, y$ momenta except if the momenta are 0 , in which case we do nothing. With these new momenta, we form the inverted partitions $\lambda_{i}^{\text {inv }}, \lambda_{j}^{\text {inv }}$ and check whether these violate the admissibility rule, i.e., whether $\left|\lambda_{i}^{\text {inv }}-\lambda_{j}^{\text {inv }}\right|<3$. We then have to analyze seven cases separately out of which three cases turn out to be problematic:

(1) $k_{x i} \neq 0, \quad k_{y i} \neq 0, \quad k_{x j} \neq 0, \quad k_{y j} \neq 0, \quad \lambda_{i}^{\text {inv }}=$ $N_{x} N_{y}+N_{x}-\lambda_{i}, \quad \lambda_{j}^{\text {inv }}=N_{x} N_{y}+N_{x}-\lambda_{j}, \quad$ and $\left|\lambda_{i}^{\text {inv }}-\lambda_{j}^{\text {inv }}\right|=\left|\lambda_{i}-\lambda_{j}\right|>3$-inversion symmetry preserved.

(2) $k_{x i}=0, \quad k_{y i} \neq 0, \quad k_{x j} \neq 0, \quad k_{y j} \neq 0, \quad \lambda_{i}^{\text {inv }}=$ $N_{x} N_{y}-\lambda_{i}, \quad \lambda_{j}^{\text {inv }}=N_{x} N_{y}+N_{x}-\lambda_{j}, \quad$ and $\left|\lambda_{i}^{\text {inv }}-\lambda_{j}^{\text {inv }}\right|=\left|N_{x}+\lambda_{i}-\lambda_{j}\right|$-and hence inversion symmetry is not preserved in the cases, $k_{y j}=k_{y i}$, $k_{x j}=N_{x}-2, N_{x}-1$, or $k_{y j}=k_{y i}+1, k_{x j}=1,2$.

(3) $k_{x i}=0, \quad k_{y i} \neq 0, \quad k_{x j} \neq 0, \quad k_{y j}=0, \quad \lambda_{i}^{\text {inv }}=$ $N_{x} N_{y}-\lambda_{i}, \quad \lambda_{j}^{\text {inv }}=N_{x}-\lambda_{j}, \quad$ and $\quad\left|\lambda_{i}^{\text {inv }}-\lambda_{j}^{\text {inv }}\right|=$ $\left|N_{x} N_{y}-N_{x}-\lambda_{i}+\lambda_{j}\right|$-and hence inversion symmetry is not preserved in the cases, $k_{y i}=N_{y}-1$, $k_{x j}=1,2$.

(4) $k_{x i}=0, \quad k_{y i} \neq 0, \quad k_{x j}=0, \quad k_{y j} \neq 0, \quad \lambda_{i}^{\text {inv }}=$ $N_{x} N_{y}-\lambda_{i}, \quad \lambda_{j}^{\text {inv }}=N_{x} N_{y}-\lambda_{j}, \quad$ and $\left|\lambda_{i}^{\text {inv }}-\lambda_{j}^{\text {inv }}\right|=$ $\left|\lambda_{i}-\lambda_{j}\right|>3$-inversion symmetry preserved.

(5) $k_{x i}=0, k_{y i}=0, k_{x j} \neq 0, k_{y j} \neq 0, \lambda_{i}^{\text {inv }}=\lambda_{i}=0$, $\lambda_{j}^{\text {inv }}=N_{x} N_{y}+N_{x}-\lambda_{j}, \quad$ and $\quad\left|\lambda_{i}^{\text {inv }}-\lambda_{j}^{\text {inv }}\right|=$ $\left|N_{y} N_{x}+N_{x}+\lambda_{i}-\lambda_{j}\right|>3$ if $N_{x}>1$-inversion symmetry preserved.
(6) $k_{x i}=0, k_{y i}=0, k_{x j} \neq 0, k_{y j}=0, \lambda_{i}^{\text {inv }}=\lambda_{i}=0$, $\lambda_{j}^{\text {inv }}=N_{x}-\lambda_{j}$, and $\left|\lambda_{i}^{\text {inv }}-\lambda_{j}^{\text {inv }}\right|<3$-for $\lambda_{j}=$ $k_{x j}=N_{x}-2, N_{x}-1$, then inversion symmetry is not preserved.

(7) $k_{x i}=0, \quad k_{y i}=0, \quad k_{x j}=0, \quad k_{y j} \neq 0, \quad \lambda_{i}^{\text {inv }}=$ $\lambda_{i}=0, \quad \lambda_{j}^{\text {inv }}=N_{x} N_{y}-\lambda_{j}, \quad$ and $\left|\lambda_{i}^{\text {inv }}-\lambda_{j}^{\text {inv }}\right|=$ $\left|N_{x} N_{y}-\lambda_{j}\right|>3$-inversion symmetry preserved.

Despite the above, in most (but not all) of the cases, the counting of states per momentum is still given by the $(1,3)$ partitions of the same total momentum $K_{x}=\sum_{i=1}^{N} k_{x i}$ and $K_{y}=\sum_{i=1}^{N} k_{y i}$, even though there is no inversion symmetry in the partitions (as proven above; we stress that the total counting of quasiholes always matches that of $(1,3)$ partitions, as it should for a Laughlin state; only the 2D-to1D mapping proposed in this paper sometimes fails for quasihole states). What happens in these cases is that momentum sectors related by inversion symmetry both contain the same number of partitions, which, under the inversion operation, are not $(1,3)$ admissible. So the effect of the 2D-to-1D mapping on the sectors not respecting the inversion symmetry is magically canceled. We find that the matching per momentum sector of the exact diagonalization states and the (1,3)-admissible partitions to be a rather remarkable demonstration of the $\nu=1 / 3$ character of the states.

[1] C. L. Kane and E. J. Mele, Quantum Spin Hall effect in Graphene, Phys. Rev. Lett. 95, 226801 (2005).

[2] B. Andrei Bernevig, Taylor L. Hughes, and Shou-Cheng Zhang, Quantum Spin Fall Effect and Topological Phase Transition in HgTe Quantum Wells, Science 314, 1757 (2006).

[3] M. Koenig, S. Wiedmann, C. Brune, A. Roth, H. Buhmann, L. W. Molenkamp, X.-L. Qi, and S.-C. Zhang, Quantum Spin Hall Insulator State in HgTe Quantum Wells, Science 318, 766 (2007).

[4] D. Hsieh, D. Qian, L. Wray, Y. Xia, Y. S. Hor, R. J. Cava, and M. Z. Hasan, A Topological Dirac Insulator in a Quantum Spin Hall Phase, Nature (London) 452, 970 (2008).

[5] D. A. Pesin and L. Balents, Mott Physics and Band Topology in Materials with Strong Spin-Orbit Interaction, Nature Phys. 6, 376 (2010).

[6] S. Rachel and K. Le Hur, Topological Insulators and Mott Physics from the Hubbard Interaction, Phys. Rev. B 82, 075106 (2010).

[7] D. N. Sheng, Z.-C. Gu, K. Sun, and L. Sheng, Fractional Quantum Hall Effect in the Absence of Landau Levels, Nature Commun. 2, 389 (2011).

[8] T. Neupert, L. Santos, C. Chamon, and C. Mudry, Fractional Quantum Hall States at Zero Magnetic Field, Phys. Rev. Lett. 106, 236804 (2011).

[9] Y.-F. Wang, Z.-C. Gu, C.-D. Gong, and D. N. Sheng, Fractional Quantum Hall Effect of Hard-Core Bosons in Topological Flat Bands, Phys. Rev. Lett. 107, 146803 (2011). 
[10] E. Tang, J.-W. Mei, and X.-G. Wen, High-Temperature Fractional Quantum Hall States, Phys. Rev. Lett. 106, 236802 (2011).

[11] F.D.M. Haldane, Model for a Quantum Hall Effect Without Landau Levels: Condensed-Matter Realization of the "Parity Anomaly," Phys. Rev. Lett. 61, 2015 (1988).

[12] K. Sun, Z. Gu, H. Katsura, and S. Das Sarma, Nearly-Flat Bands with Nontrivial Topology, Phys. Rev. Lett. 106, 236803 (2011).

[13] A. Kol and N. Read, Fractional Quantum Hall Effect in a Periodic Potential, Phys. Rev. B 48, 8890 (1993).

[14] G. Möller and N. R Cooper, Composite Fermion Theory for Bosonic Quantum Hall States on Lattices, Phys. Rev. Lett. 103, 105303 (2009).

[15] G. Möller and N. R Cooper, Condensed Ground States of Frustrated Bose-Hubbard Models, Phys. Rev. A 82, 063625 (2010).

[16] B. Andrei Bernevig and F. D. M. Haldane, Model Fractional Quantum Hall States and Jack Polynomials, Phys. Rev. Lett. 100, 246802 (2008).

[17] N. Regnault and B. A. Bernevig, Emergent Many-Body Translational Symmetries of Abelian and Non-Abelian Fractionally Filled Topological Insulators, arXiv:1110.4488.
[18] E. J. Bergholtz, J. Kailasvuori, E. Wikberg, T. H. Hansson, and A. Karlhede, The Pfaffian Quantum Hall State Made Simple-Multiple Vacua and Domain Walls on a Thin Torus, Phys. Rev. B 74, 081308 (2006).

[19] A. Vaezi, Fractional Quantum Hall Effect at Zero Magnetic Field, arXiv:1105.0406.

[20] X.-L. Qi, Generic Wave-Function Description of Fractional Quantum Anomalous Hall States and Fractional Topological Insulators, Phys. Rev. Lett. 107, 126803 (2011).

[21] H. Li and F. D. M. Haldane, Entanglement Spectrum as a Generalization of Entanglement Entropy: Identification of Topological Order in Non-Abelian Fractional Quantum Hall Effect States, Phys. Rev. Lett. 101, 010504 (2008).

[22] A. Sterdyniak, N. Regnault, and B. A. Bernevig, Extracting Excitations from Model State Entanglement, Phys. Rev. Lett. 106, 100405 (2011).

[23] M. Hermanns, A. Chandran, N. Regnault, and B. A. Bernevig, Haldane Statistics in the Finite Size Entanglement Spectra of Laughlin States, Phys. Rev. B 84, 121309(R) (2011).

[24] R. Thomale, A. Sterdyniak, N. Regnault, and B. A. Bernevig, Entanglement Gap and a New Principle of Adiabatic Continuity, Phys. Rev. Lett. 104, 180502 (2010). 